\title{
Performance and First Science Observations with ARGOS
}

\author{
Sebastian Rabien*a, Marco Bonaglia ${ }^{\mathrm{c}}$, José Luis Borelli ${ }^{\mathrm{b}}$, Peter Buschkamp ${ }^{\mathrm{a}}$, Lorenzo Busonic, \\ Andrew Cardwel ${ }^{\mathrm{f}}$, Matthias Deysenroth ${ }^{\mathrm{a}}$, Simone Esposito ${ }^{\mathrm{c}}$, Wolfgang Gässler ${ }^{\mathrm{b}}$ Hans Gemperlein $^{\mathrm{a}}$, \\ Iskren Georgiev $^{\mathrm{b}}$, Christoph Kohlmann ${ }^{\mathrm{a}}$, Martin Kulas ${ }^{\mathrm{b}}$, Michael Lefebvre ${ }^{\mathrm{f}}$, Tommaso Mazzonic, \\ Gilles Orban de Xivry ${ }^{\mathrm{a}}$, Diethard Peter ${ }^{\mathrm{b}}$, Alfio Puglisi ${ }^{\mathrm{c}}$, Walfried Raab ${ }^{\mathrm{a}}$, Gustavo Rahmer ${ }^{\mathrm{f}}$, Matthias \\ Rosensteiner ${ }^{\mathrm{a}}$, Alexander Sivitili ${ }^{\mathrm{b}}$, Julian Ziegleder ${ }^{\mathrm{a}}$
}

\author{
${ }^{a}$ Max-Planck-Institut für extraterrestrische Physik, Garching, Germany \\ ${ }^{\mathrm{b}}$ Max-Planck-Institut für Astronomie, Heidelberg, Germany \\ ${ }^{\mathrm{C}} \mathrm{INAF}$ - Osservatorio Astrofisico di Arcetri \\ ${ }^{\mathrm{f}}$ Large Binocular Telescope Observatory
}

\begin{abstract}
Coming online for scientific operations, the ARGOS facility is boosting the imaging and spectroscopic capabilities at the LBT. With six Rayleigh laser guide stars and the corresponding wavefront sensing, ARGOS corrects the ground layer distortions for both LBT $8.4 \mathrm{~m}$ telescopes with its adaptive secondary mirrors. Under most conditions this setup delivers a PSF size reduction by a factor $\sim 2-3$. With the two LUCI infrared imaging and MOS spectroscopy instruments receiving the corrected images, observations in the near infrared can be performed at high spatial and spectral resolution. We will briefly discuss the final ARGOS technical setup and the adaptive optics performances. With first scientific observations been conducted, we will show that imaging cases with GLAO are nicely boosting several science programs from cluster CMD, Milky Way embedded star formation and Cepheids, BHs in nearby galaxies to extragalactic deep fields. In the unique combination of ARGOS with the multi-object NIR spectroscopy available in LUCI, first scientific observations have been performed on local and high-z objects. Those high spatial and spectral resolution observations nicely demonstrate the capabilities now at hand with ARGOS at the LBT. Inhere we describe briefly the system and show examples of science observations from nearby clusters to high redshift gravitationally lensed objects.
\end{abstract}

Keywords: Laser guide star, ground layer adaptive optics, large binocular telescope

\section{INTRODUCTION}

The Advanced Rayleigh Guided Ground layer adaptive Optics System (ARGOS) has been designed to deliver a ground layer adaptive optics correction to both of LBTs eyes- shrinking the PSF size by a factor 2-3. The primarily goal of ARGOS is to enhance the image quality and the spectroscopic efficiency of the twin multi-object spectrometers LUCI1 and LUCI2. [1] Enhancing the image quality with ground layer adaptive optics has several advantages: The spatial resolution gain gives insights into details of an objects structure. But apart from this obvious gain the signal to noise in spectroscopy benefits strongly from sharpening the image. With the required integration time to reach a given signal to noise ratio being inversely proportional to the square PSF size, we will be able to carry out observations in much shorter time. In addition the spectroscopic slit width can be decreased accordingly, reducing the sky background and enhancing the spectral resolution.

Additionally, the gain of the ground layer adaptive optics correction is available over the full field of view of the LUCI instruments, enabling science cases to benefit from AO that cannot be done with single conjugated systems.

*srabien@mpe.mpg.de 


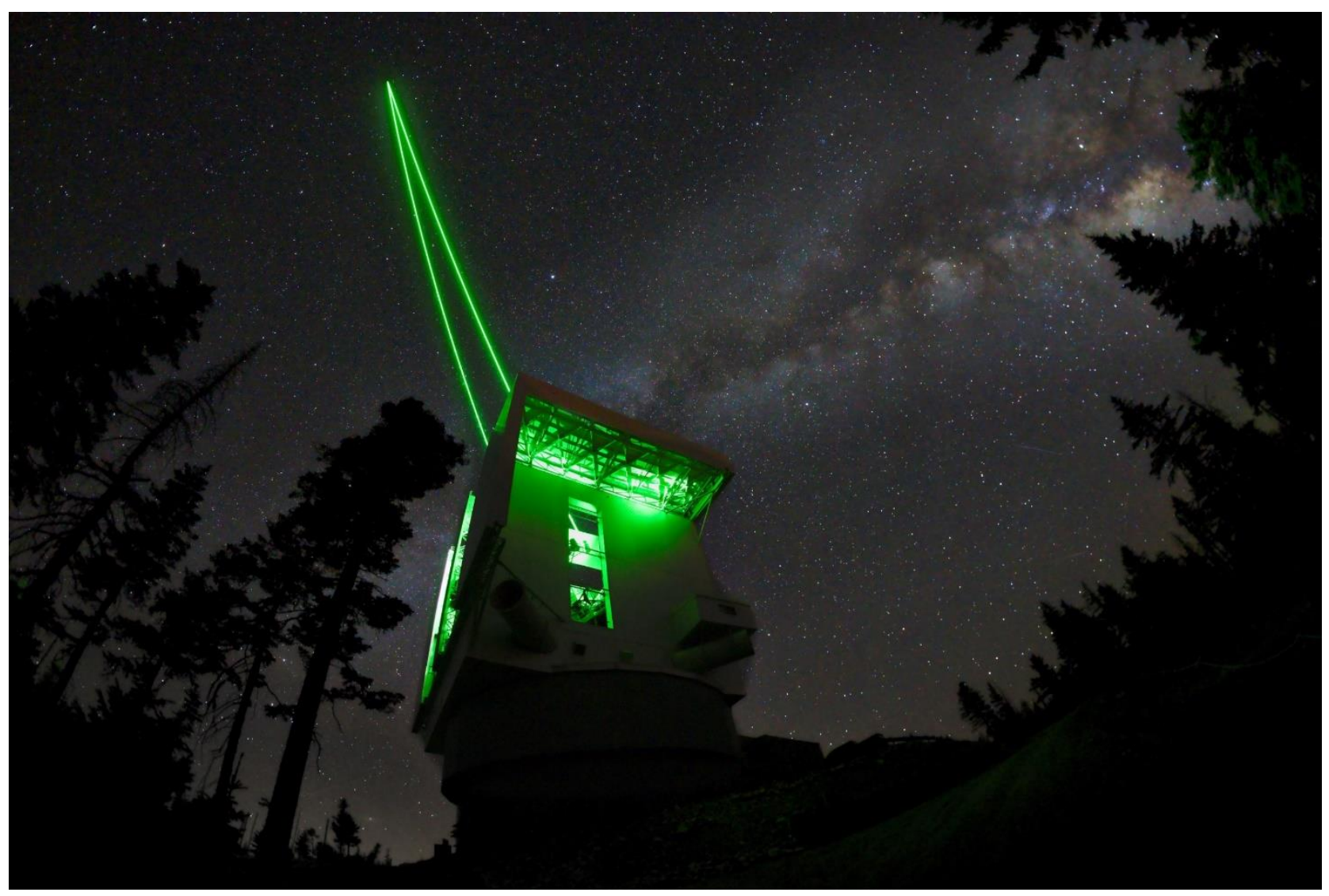

Figure 1: The large binocular telescope with the ARGOS system propagating a bundle of laser beams on each side of the telescope to sky. Each visible beam consists of three individual laser beams forming the constellation of guide stars in the atmosphere. The pulsed Nd:YAG lasers are triggered at $10 \mathrm{kHz}$, with the wavefront sensors adjusted to receive the scattered light from a $12 \mathrm{~km}$ distance above the telescope.

To summarize, ARGOS with LUCI at the LBT offers:

- Observations with two $8.4 \mathrm{~m}$ telescopes in binocular mode at the same time.

- GLAO correction with a 0.2-0.3 arcsec resolution over two times $4 \times 4$ arcmin field of view.

- A fairly homogeneous PSF shape over this full field.

- A large field selection of $2 \times 3$ arcmin for the NGS tilt star, selectable up to $18 \mathrm{mag}$.

- NIR imaging of the full field.

- GLAO corrected NIR spectroscopy with the LUCI multi object spectrograph and custom cut slit masks.

The last bullet really emphasizes one of the unique capabilities that ARGOS allows for. Currently this combination is only available at the LBT, enabling spectroscopic observations at high spatial and spectral resolution. The high spatial resolution allows the spectroscopic slits to be cut as small as $0.25-0.3$ ', wide, pushing the spectral resolution up to R 10000, enabling the detection of fine structures in the velocity distribution of e.g. high-z galaxies.

The ARGOS system uses a constellation of three guide stars above each of the LBT mirrors. The laser beacons are created with Rayleigh scattering of high power green beams. These 'Rayleigh laser guide stars' are based on industrial style laser units, powerful enough to create the guide stars with a high photon flux for the wavefront sensing. When running the sensors at a $1 \mathrm{kHz}$ rate we are still counting up to $\sim 1500$ photons per subaperture. Over the full extend of the commissioning 
runs we have never seen cases when the photon flux from the laser beacons has been too low. Figure 1 shows the ARGOS binocular laser beams when propagated to sky.

The direct benefits that ARGOS will bring to the LBT are afforded by the factor of 2-3 improvement in the spatial resolution. Indeed, ARGOS can be considered as a 'seeing enhancer' for the existing facility instruments that enables one to boost several science cases. This is because:

- observations can be done much faster, saving a significant amount of observing time.

- demanding science programmes, that would normally require the best seeing conditions, can instead be carried out during most nights.

- observations of fields can be conducted where standard AO is impossible due to the NGS star being too faint or too far.

In contrast to single conjugated adaptive optics programs, ARGOS aims to provide enhanced resolution and sensitivity for both imaging and multi-object spectroscopy over a very wide field of view. A wide range of science will strongly benefit by the enhanced resolution and ensquared energy that ARGOS delivers. Amongst others, the scientific topics that can be addressed with the aid of GLAO span a wide range from extragalactic cases such as high-z galaxy dynamics, AGN and QSO host galaxies, to Galactic astrophysical questions about planets, cepheids or stellar clusters. With the GLAO system a major gain in resolution over a wide seeing range can be achieved, thus decreasing integration times required by large factors.

In the following we give a brief introduction into the ARGOS technical system and an overview of the LUCI capabilities, followed by some examples of observations that have been done during commissioning of the system.

\section{SYSTEM DESCRIPTION}

The main units of ARGOS are: The laser systems, delivering the laser beams; the launch system expanding the lasers, propagate them to sky and focus to the appropriate height. The wavefront sensing units receive the beacons, measure the ground layer turbulence and sends the correction signal to the deformable secondary mirrors. In addition, the first light AO pyramid sensors [5] receive the light from natural guide stars, measure the tilt and slowly serve as truth sensing modules. In addition many more subunits for general services, the instrument and real time control, an airplane detection system and laser safety controls are present. More details of the system have already been described in [2][3][4]. In the following we give a short introduction to the laser and the wavefront sensor units.

\section{Laser \& launch systems}

For each of the LBTs eyes there is an ARGOS laser system mounted to deliver the laser constellations (see Figure 2). Each of the 'laser boxes' provides a sealed and temperature controlled environment for the three laser units per side and the according beam steering and shaping optics. The lasers themselves are pulsed, frequency doubled Nd:YAG lasers produced by Innolas $\mathrm{GmbH}$. Each of the units is specified to emit $18 \mathrm{~W}$ at a $10 \mathrm{kHz}$ rate with $\sim 40 \mathrm{~ns}$ pulse width. The total power over time of each laser head varied slightly over operation and commissioning duration, resulting in 14-18W being available for the guide star creation and unit laser head. The beams are diffraction limited with an $\mathrm{M}^{2}$ better than 1.2. In the beam train after the laser exit, the lasers are expanded to a $\sim 6 \mathrm{~mm}$ beam and folded by two periscope mirrors into the proper constellation direction. Cameras that monitor the field and the pupil location of the beams are used to control the locations automatically. 

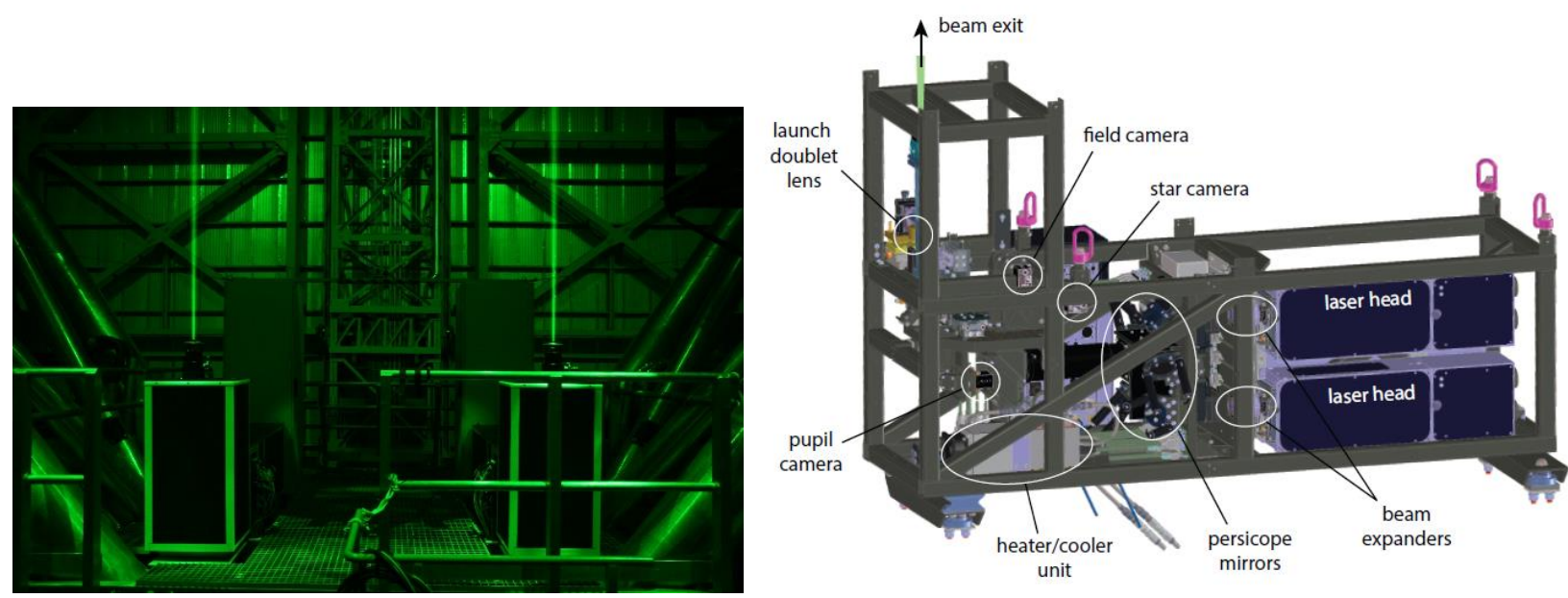

Figure 2: On the left a photograph of the two Argos laser systems mounted into the LBT structure. The green laser beams can be seen exiting the boxes before the protective covers have been mounted. Right: CAD model of the laser box without cover.

Inside the laser box the three beams join at a common 'pupil' location, where a fast piezo driven tip-tilt mirror is located. This mirror is used to control common position jitter introduced by the launch system vibrations. This unit proved extremely valuable for the final performance, removing the windshake and vibrations, with a system detecting the accelerations of the launch mirrors and the position jitter on the wavefront sensor [6]. After this mirror the beams are directed to the first launch system input lens for beam focusing, followed by a tilted plate astigmatism compensator. A 400mm aspheric lens and two large fold mirrors finally complete the launch system and direct and focus the laser constellation to sky. There is another multitude of controls and beam diagnostics hardware in the laser and launch system: like power measurement, pulse diagnostics, collimation testing, environment control and launch mirror shape and thermal control.

\subsection{Wavefront sensing}

Both ARGOS LGS wavefront sensors are based on a Shack Hartmann (SH) scheme to detect the laser guide stars Figure 3 shows a drawing and the SH pattern on the detector. Details are described in [9]. Being located in the plane of the $12 \mathrm{~km}$ focus, a field stop with $\sim 4.5$ arcsec diameter forms the entrance to the WFS optics. For each of the guide stars, a collimating lens re-images the pupil over a periscope onto the entrance of the Pockels cells. The periscope mirrors do bring the constellation closer together, and as well allow for field stabilization in the sensor.

In the collimated beam -being strictly collimated only when the laser pulse is exactly at $12 \mathrm{~km}$ distance- a Pockels Cell assembly is located. This unit acts as an optical shutter, opening $80 \mu \mathrm{s}$ after the laser pulse has been send to sky and closing again $2 \mu \mathrm{s}$ later. This lets only those photons pass towards the CCD that have been scattered between 12 and $12.3 \mathrm{~km}$ above the telescope. With the lasers repetition rate being set to $10 \mathrm{kHz}$, we accumulate the charge of ten pulses on the sensor before the readout is triggered. After the gating units each beam passes a focusing lens and a common collimator that form a pupil image on a single lenslet array directly before the CCD. The optics is adjusted such that the three SH patterns are nicely distributed over the CCD, each sub-aperture owns 8x8 pixels and the split frame transfer does not cross subapertures. More information on the wavefront sensor performance is given in [9].

The CCD itself is based on a deep- depletion technology, offers 264x264 pixels and a low readout noise at a kHz frame rate. Details can be found in [8]. 


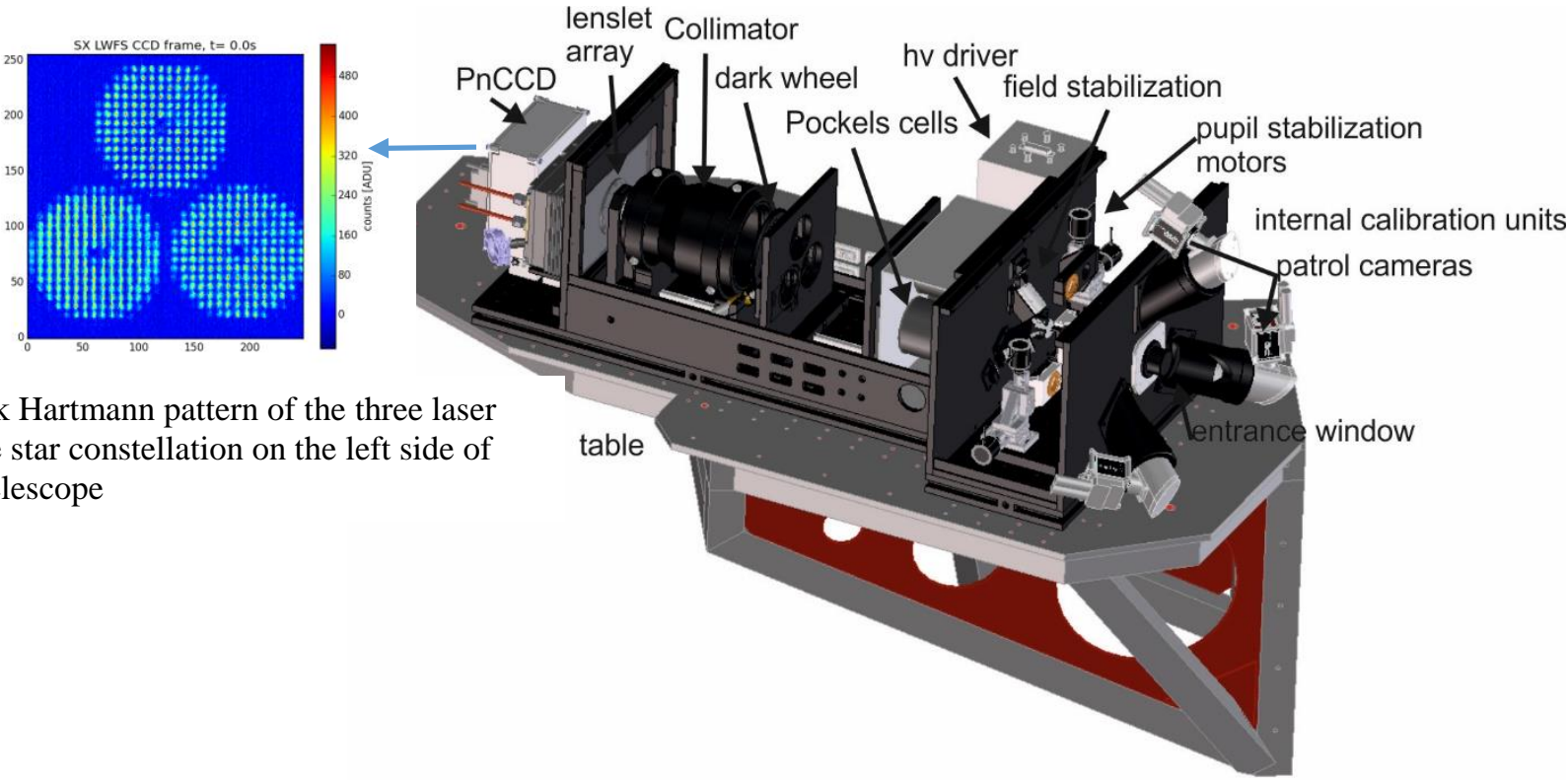

Figure 3: Drawing of one of the ARGOS wavefront sensors. The light from the three laser guide stars is coming from the right side and enters the sensor through a field stop. Patrol cameras with an 1 arcmin field of view aid the acquisition of the LGS. A first collimator and periscope is actively used in closed loop to control the field and pupil position on the CCD. The three Pockels cells gate the desired light column out of the backscattered signal. The collimator sends the light onto a common lenslet array and finally onto the CCD where the Shack Hartmann pattern of the laser beacon constellation is seen.

\section{The LUCI spectrographs}

Receiving the GLAO corrected science photons, the LUCI1 and LUCI2 instruments are the 'customers' of ARGOS. These two identical instruments are designed for imaging and spectroscopy in the near infrared. In short, the LUCIs can perform imaging in the broad $\mathrm{z}, \mathrm{J}, \mathrm{H}, \mathrm{Ks}, \mathrm{K}$ bands and a number of narrow band filters. In spectroscopic mode a cryogenically exchangeable mask in the image plane allows to select the object, either with long slits, or with custom cut multi-object masks. Since the laser cutting of the masks opens up quite some freedom in designing those, the slit width and as well the shape can be adopted to the needs of the science to be done. A suite of selectable gratings sets the spectral- and the choice of the camera the spatial resolution. In typical seeing limited observations, the slit width would be chosen to 1" width, to allow all light from the blurred image to enter the slit. With the matched F\#1.8 camera the resulting spectrum on the detector gives an $\sim \mathrm{R}=3000$ in $\mathrm{H}$ and 2500 in $\mathrm{K}$. In spatial direction the pixelscale amounts to 0.25 "'/pix with this camera, and the resolution of course is dictated by the seeing. With choosing the higher resolution camera F\#3.75 and a smaller slit width of 0.25 " to 0.3 " the spectral resolution is boosted up to $\mathrm{R}=12000$ to $\mathrm{R}=10000$, and the spatial axis can be sampled with $0.12 \%$ /pix. This high resolution mode is typically chosen when observing with ARGOS. A photograph of both LUCI vacuum vessels from the top is shown in Figure 4. 


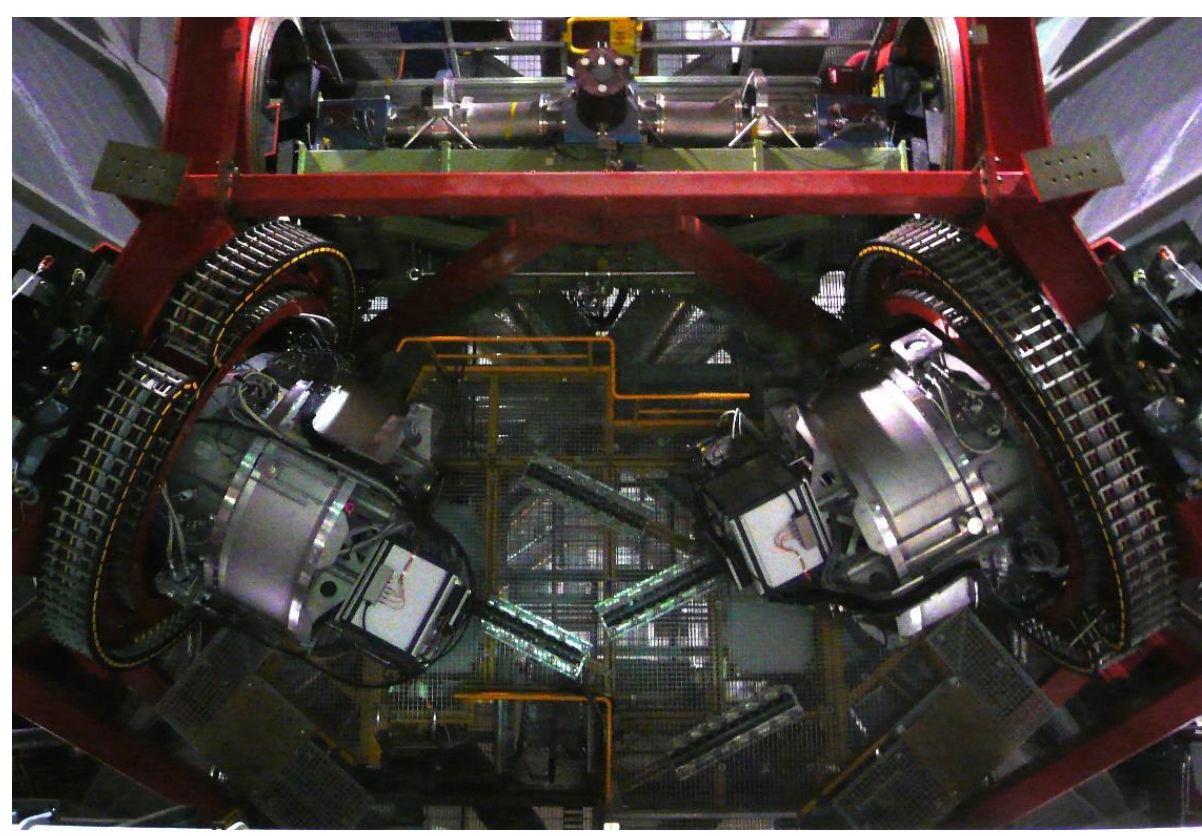

Figure 4: Photograph of the two LUCI instruments as seen from top at the telescope. Both of the large cryogenic NIR spectrographs receive the light in the bent Gregorian focus from each of the $8.4 \mathrm{~m}$ LBT mirrors.

\section{ON SKY PERFORMANCE}

Over the extend of the years 2015 to 2017 ARGOS has seen approximately 90 nights of commissioning. During those nights we have set up and tested the system from earliest laser on sky tests to full closed loop operation. Commissioning and AO results can be found in [10][11][12].Now, at the end of 2017 we do see a system that is capable of conducting science operation feeding the two sides of LBT simultaneously with laser guide star corrected light. The capability of doing now full binocular operation with ARGOS and two LUCIs is a real boost for the science outcome. Having two $8.4 \mathrm{~m}$ telescopes available at the same time with a GLAO corrected PSF of 0.2 " to 0.3 " in size is a massive benefit for plenty of science cases. On one hand the PSF size compares well with HST data, where now ARGOS can complement imaging taken with HST with a K-band observation at similar resolution. For e.g. constraining a galaxy's SED the K-band flux is really crucial. From the many observations that have been conducted already during the commissioning of the system, we pick cases for this proceedings that highlight the capabilities of a ground layer adaptive optics system in combination with the NIR imaging and spectroscopy.

Figure 5 shows the image of NGC2419, an old cluster of stars in the halo of the Milky Way. NGC 2419 is particularly interesting for its large distance $(90 \mathrm{kpc})$ to the Milky Way. For that NGC2419 is an ideal probe of the Galactic potential and it contains almost 80 RRLyrae stars with known periods. While precise proper motions may be obtained from HST or perhaps with future Gaia data release, the most precise distances in the Galactic halo can only be measured for RRLyrae stars. In the Ks-band, for example, the period-luminosity relation for RRLyrae stars can provide as precise distances as 1$2 \%$. In that respect NGC2419 has been visited during the ARGOS commissioning several times. Apart from the scientific insight in the galactic potential and the cluster CMD that can be gained with the measurements, the cluster provides us also with nicely distributed stars as PSF probes for the adaptive optics performance.

Figure 6 shows a timeline of a typical observation that has been carried out on this cluster. Over approximately one hour multiple images of the cluster are taken with the J, H, and Ks filters. Due to the crowded field sky frames are required for the observation. When moving to 'sky' we typically do open the adaptive optics loop, since we usually loose the tilt star, and may be out of the satellite target window. Those frames nicely provide the open loop comparison images to track the adaptive optics performance, while no time is lost for science. In Figure 6 the red dots mark the FWHM measured in the open loop sky frames, while the blue dots show the FWHM in closed loop science images. That observation, carried out at 1 " to 1.4 ' DIMM optical seeing, results in 0.3 ' to 0.4 ' ' performance in J and 0.25 ' to 0.3 ', in Ks, reducing the natural seeing nicely by a factor 2 to 3 . 

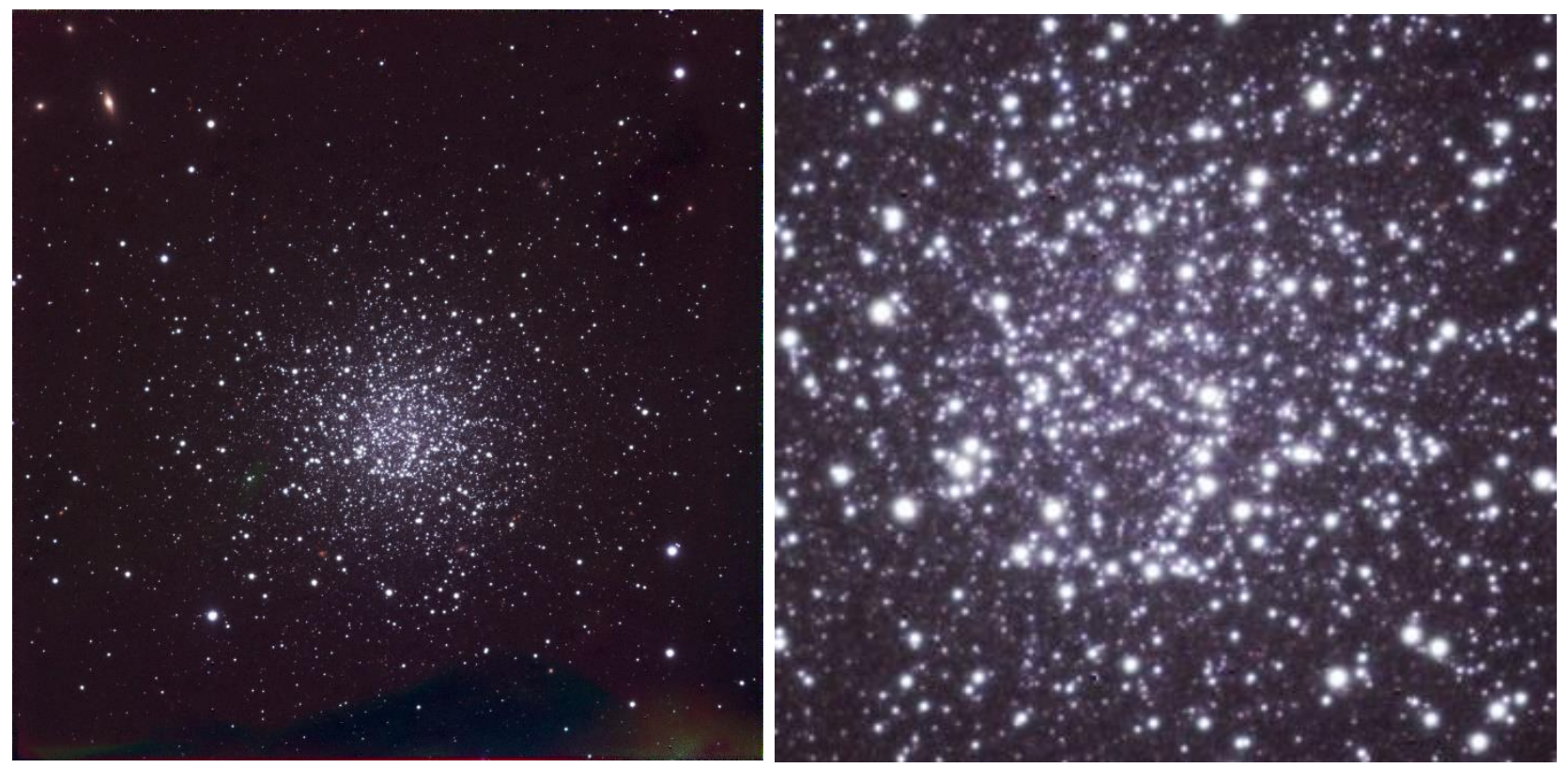

Figure 5: JHKs color composite image of NGC2419. Left the full $4 \times 4$ arcmin field. Right a zoom into the cluster core, showing the richness and density. These images where taken during commissioning of ARGOS and highlight the large field performance of the system. The large number of cluster stars provides a good map for the uniformity of the FWHM and the overall performance of the system over time.

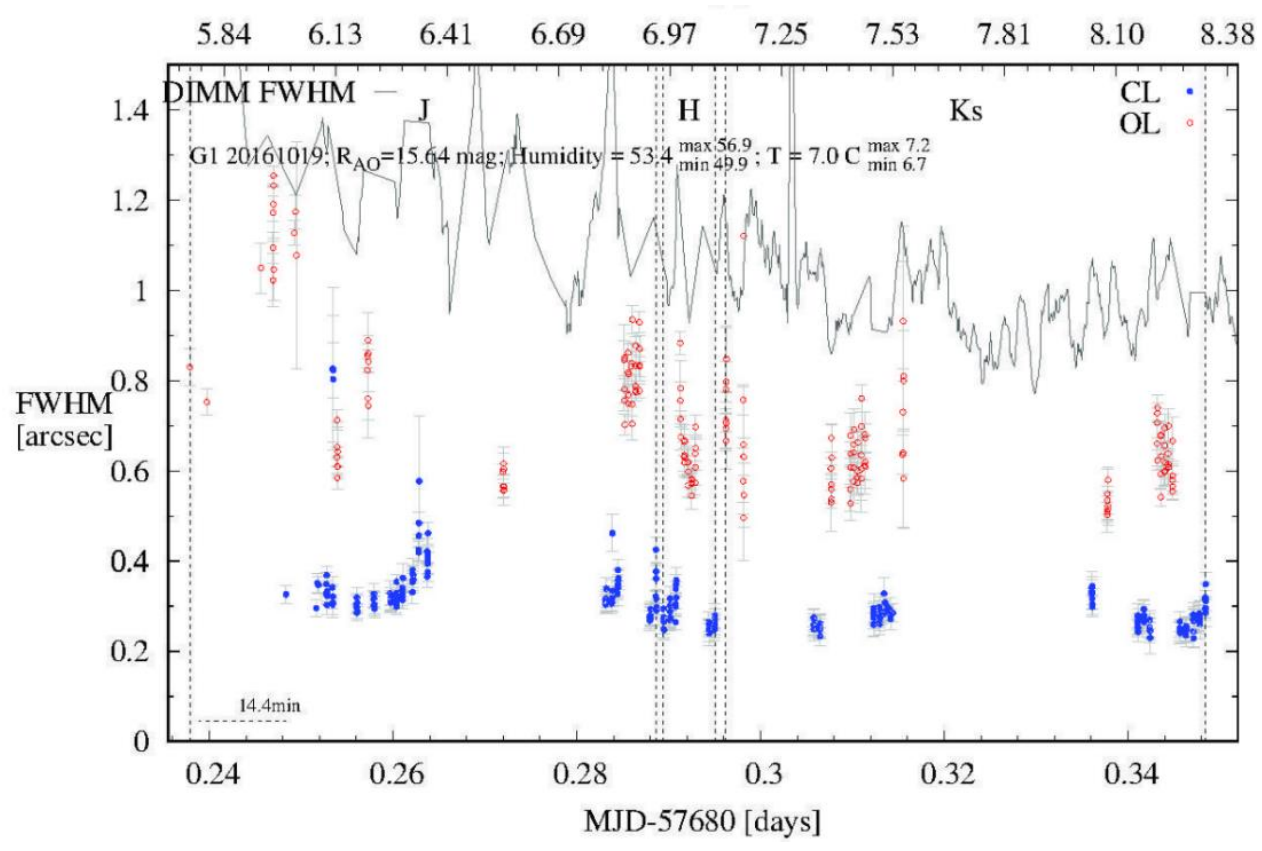

Figure 6: FWHM over time of a typical imaging observation. The observation was conducted with switching the instrument filters from $\mathrm{J}$ over $\mathrm{H}$ to $\mathrm{Ks}$ in sequence. Blue points show the measured FWHM with ARGOS in closed loop, while the red points are the FWHM in open loop, i.e. the natural seeing in the respective filter. The black line shows the optical DIMM measurements at the same time. As can be seen, the open to closed loop ratio is typically around 2-3. 

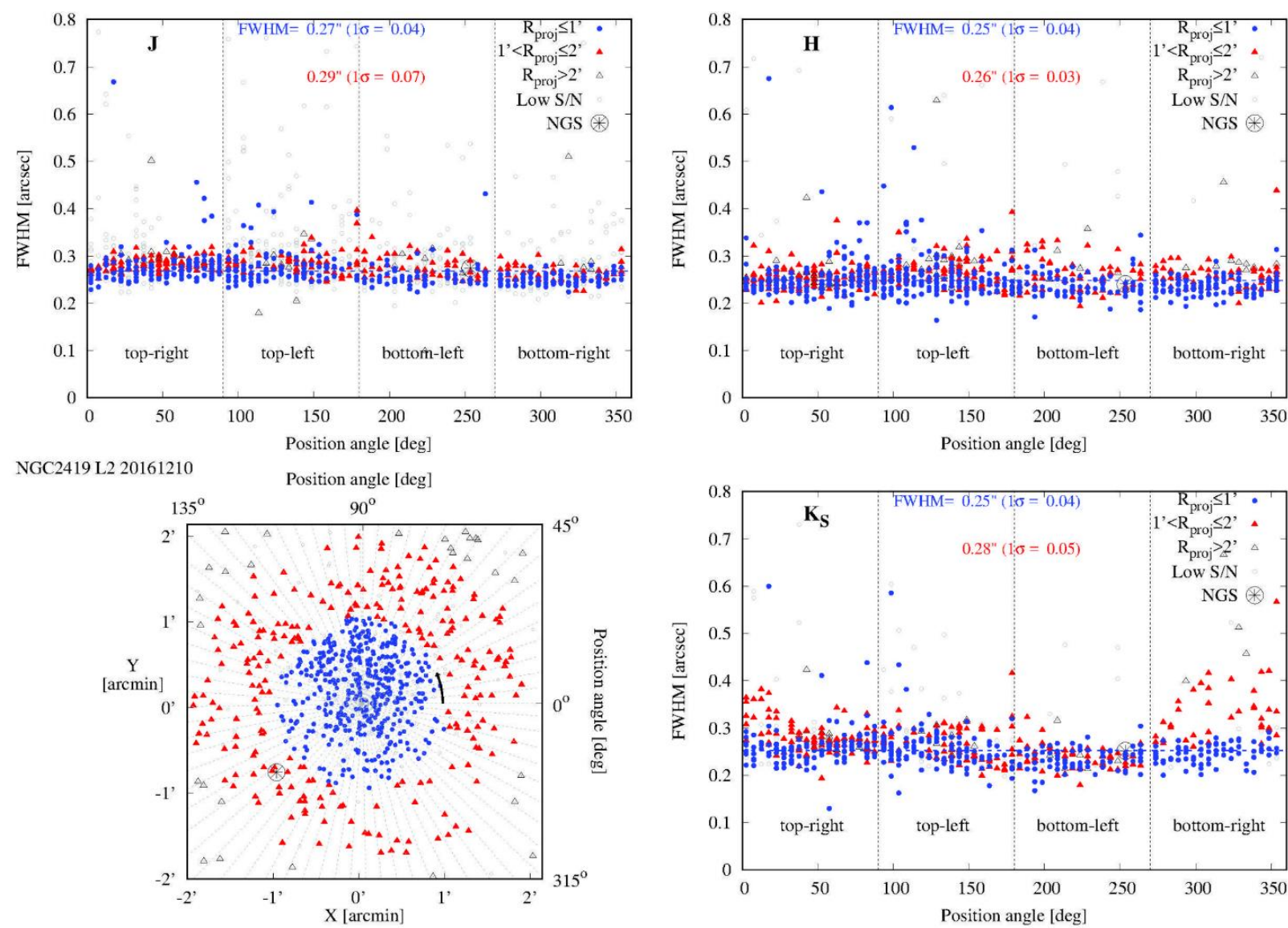

Figure 7: NGC2419 data showing the ARGOS performance over the full LUCI2 field of view. The bottom left panel shows the positions of all stars that were used to measure the azimuthal FWHM variation. Different colours (blue and red) separate stars at different radial locations, as indicated in the figure legend for each filter. With encircled (gray) asterisk is shown the location of the NGS at $\sim 225^{\circ}$. It can be seen a small $(<15 \%)$ radial increase from 0.25 " to 0.29 " of the FWHM from the centre to the detector edge. This demonstrates the high uniformity and overall stability of the PSF delivered by ARGOS.

With usual SCAO the PSF variation over the field due to the un-isoplanatism is a draw back for quite some science cases. Theoretically shown in a lot of simulations, GLAO offers a fairly homogeneously large field adaptive optics correction. With both LUCIs offering an imaging field of $4 \times 4$ arcmin, we can nicely probe the PSF uniformity in practice. Figure 7 shows measurements taken on NGC2419, testing the PSF size over the full field, and the performance in J,H,Ks. In that plot all stars within an 1 arcmin circle from the field center are noted with a blue dot, red triangles show the stars from 1 to 2 arcmin and grey the outermost ones. The location of the NGS tilt star is denoted with a grey star. Comparing the three PSF size plots for the three bands one can mainly see that there is very little PSF variation over the full field, since the Red triangles do not fall significantly above the blue 'inner' stars. One finding -seen in a lot of observations- is particular interesting. Basically the distance to the tilt star in the field does not matter on that scales. The performance is so uniform, that we can place the tilt star anywhere in the large field. This actually is a huge advantage for extragalactic science cases, very often offering only distant and sparse possibilities to select a bright natural tilt star.

Another inherent quality we recognized in the observations with LGS GLAO is the PSF stability over time. This is especially valuable for observations extending over many hours. As the natural seeing is often highly variable, the combination of lots of images over the observation period results either in being dominated by the 'bad' seeing moments, or in a selection of images, i.e. throwing away data. An example is shown in Figure 8 from an observation of IC342. The PSF extracted from the Ks-band images is nicely constant over the full observation period, while the optical seeing and the open loop data varies strongly. The reasoning behind this behavior is clearly that with ARGOS we can get rid of the ground layer turbulence contribution, being much more variable than the free atmosphere at higher altitudes. 

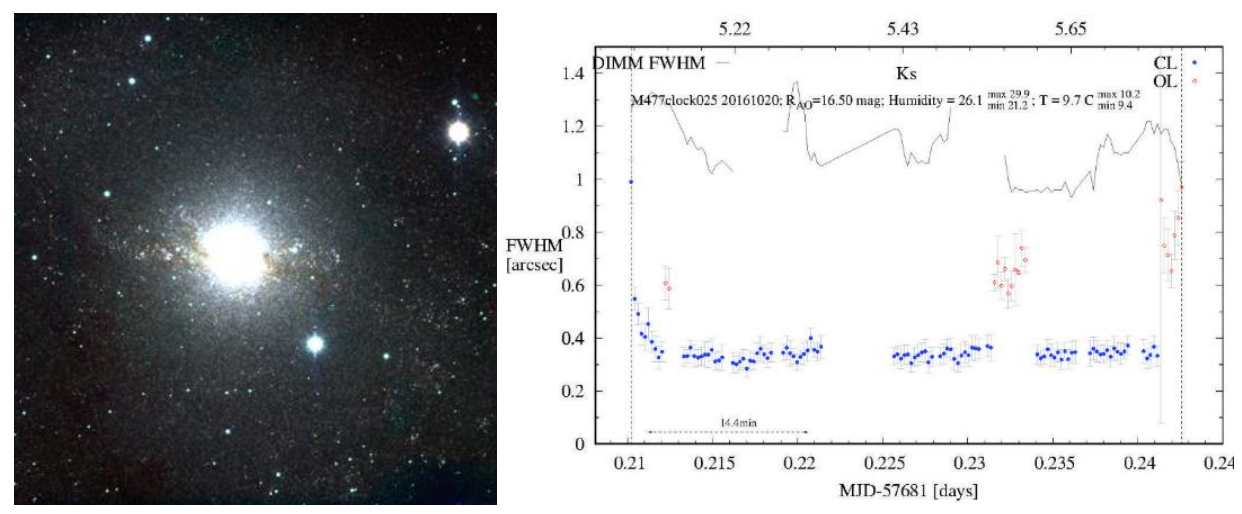

Figure 8: JHKs color composite image of IC342. Right: the PSF size extracted from each individual image over time. The first frames are dominated by the convergence of the truth sensor, after that a stable PSF of $\sim 0.3$ ', is delivered in a variable 1.2 ' seeing night.

\section{Spectroscopy with ARGOS}

One of the really unique capabilities that ARGOS offers is the combination of the ground layer adaptive optics with NIR spectroscopy. To our knowledge ARGOS plus LUCI is the only facility in the world that can deliver spectra with 0.2..0.3", spatial resolution and spectroscopy with R 10000 at the same time. Additionally the 4x4 arcmin masks for LUCI can be cut to custom shapes matching the object under study. It may be the most demanding observational setups we have tested so far during the commissioning: spectroscopy of gravitationally lensed high-z objects. Gravitational lensing offers a great opportunity to study objects at high redshift, due to the flux enhancing effect of the lens, making dim objects appear brighter and sometimes make them observable within reasonable time at all. Due to the mass distribution of the lensing clusters or massive galaxies, a lot of lensed objects appear as small and extended arcs on sky. In quite some cases these arcs extend over tens of arcseconds in length, but only over 0.2..0.3 arcsec in width. Due to the objects length small field AO IFU studies usually can only look at parts of the object, while straight slit spectrographs don't match the curvature. In that respect ARGOS plus LUCI offers an ideal match to do detailed studies of those arcs. With LUCI we can make curved matched shape slits over the full extend of the arcs, while with ARGOS we sharpen the object, such that it concentrates all the light through the narrow 0.3 " slit, to resolve velocities spatially un-smeared at $\mathrm{R}=10000$ at the proper location. As an

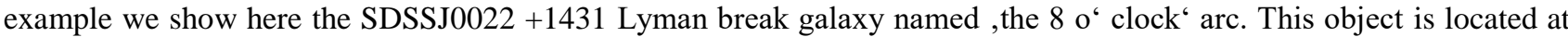
redshift of 2.73 being strongly lensed by a $z=0.38$ luminous red galaxy SDSS J002240.91+143110.4. While this object has been observed spectroscopically with SINFONI [13], NIRI [14] and Xshooter [15] already, we can draw a direct comparison with existing data. With a redshift of 2.73 this object is especially demanding to observe in H $\alpha$ emission, since it falls at $2.48 \mu \mathrm{m}$ - outside the K-band where the thermal background is already getting strong. With the ability of ARGOS plus LUCI to keep the slits small, concentrate the objects flux into the slit and have the spectral resolution high we can dig the arcs signal very well out of the massive thermal background.

In Figure 9 a sketch of such an observation is shown. Using HST, or an ARGOS pre-imaging campaign we design a mask that matches the lensed arc, and a second identical slit to allow nodding between the two. This design is sent to LBTO for the laser cutting and cryogenic insertion of the masks for the upcoming semester. Upon observation the mask is grabbed by the robot inside LUCI and inserted in the instruments focal plane. With ARGOS we nod between the slits as long as needed to reach the desired signal. The resulting spectrum of the ' 8 o clock arc' $\mathrm{H} \alpha$ emission is shown in Figure 10. Without the GLAO correction the emission is barely visible in a 5 min integration time, of course as well due to the small slit width of 0.3 '. With the adaptive optics on, the emission can be seen in a single 5 min integration. Adding now several integrations up, removing the slit curvature and wavelength calibrating gives then the final spectrum, as shown in the lowest panel of Figure 10. The spectrum shows a high level of details of the velocity distribution, dispersion and a sharp emission on the blue side of $\mathrm{H} \alpha$. This level of detail has not been seen in previous observations due to the lack of spectral resolution and signal to noise. In this proceedings we show the resulting average 1-D spectrum of the 8 o' clock arc, reaching a $\mathrm{S} / \mathrm{N}$ of $\sim 100$ within 1 h of observation. Roughly a factor of 10 better than any previous measurements. A more detailed scientific analysis of this data is pending a dedicated publication in preparation. 

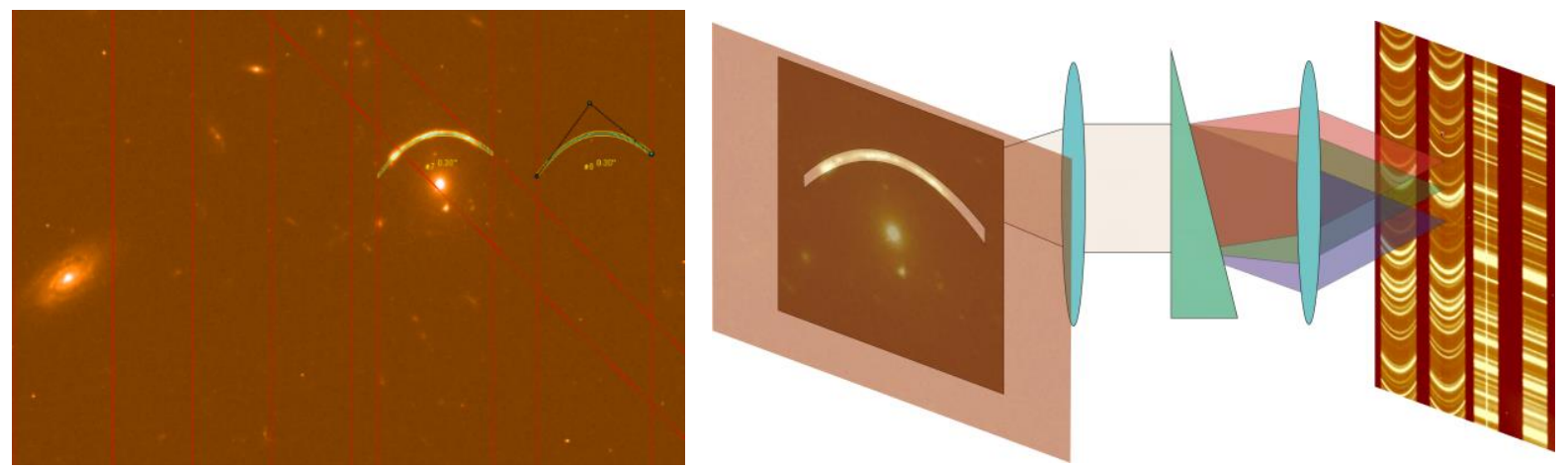

Figure 9: Basic principle of spectroscopic observations with ARGOS plus LUCI. Left: K-band an image of a gravitationally lensed object (the 8 o' clock arc) with the mask design overlaid. From the design, a custom, laser cut mask is made and inserted and stored cryogenically in the LUCI instruments. Upon observation only the light from the desired object enters the spectrometer. The ARGOS system now enables that tiny slits are used and all light from the object still can pass through. In result the sky background is low, the skylines are well separated, and the spectral resolution is high.

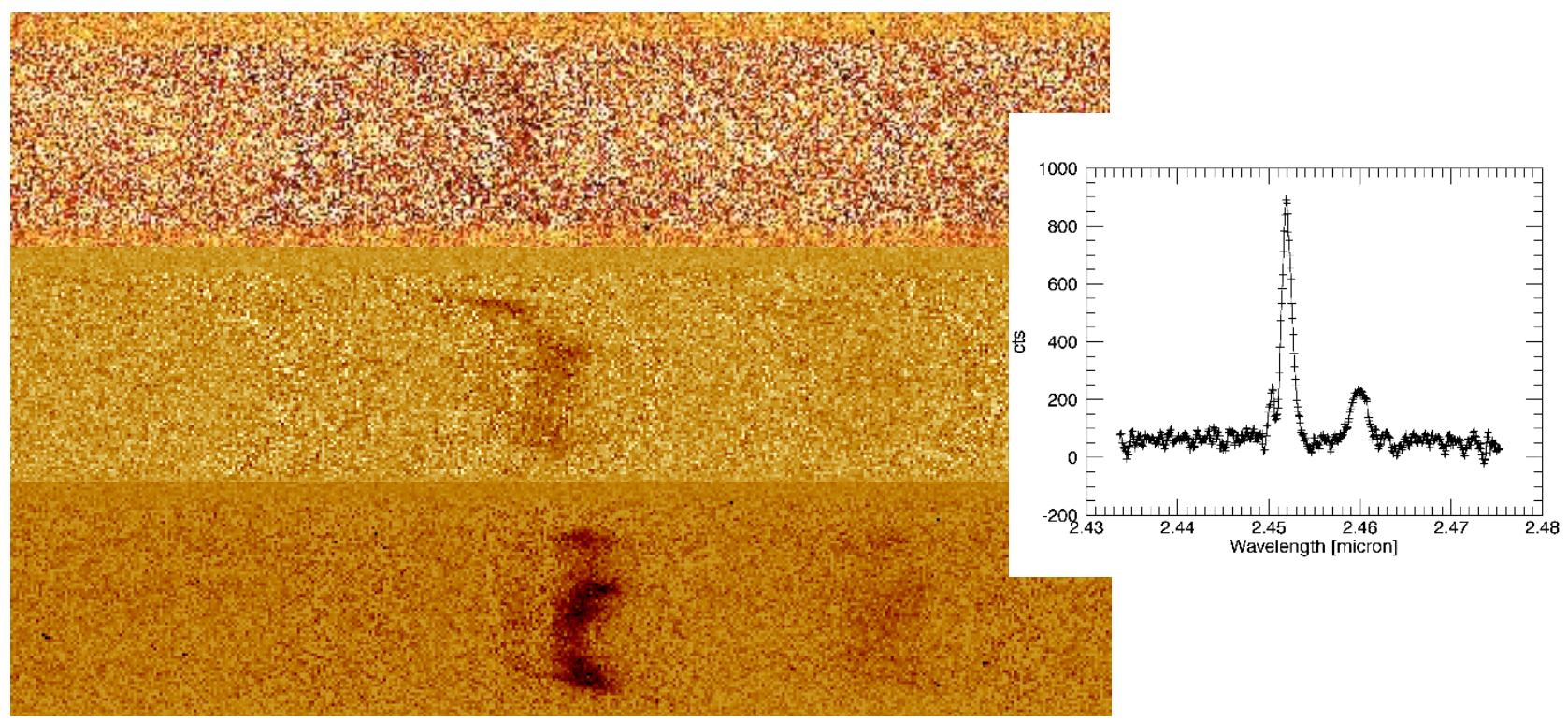

Figure 10: Observation of the $\mathrm{H} \alpha$ emission along the gravitational lensed system 8 o' clock arc. Top: a weak emission from the target in a 5 min open loop exposure. The emission is barely visible here, due to the large seeing limited PSF loosing light at the small slit. Middle: a 5 min integration with ARGOS switched on. The light from the object now nicely enters the 0.3" slit and $\mathrm{H} \alpha$ is detected easily. Bottom: Data from a combined half hour observation and correction for slit curvature. The NII emission is now nicely visible, the velocity structure can be resolved, and emission on the blue side points towards a massive outflow in this high-z galaxy. The 1-D spectrum to the right is collapsed along the spatial axis and shows the high $\mathrm{S} / \mathrm{N}$ detection in the $\mathrm{H} \alpha$ emission. Due to the spectral resolution we clearly resolve a blue sharp emission, being most likely a massive outflow from this galaxy.

While high-z observations are most demanding in terms of observation time due to the dim character of the objects, other cases usually require a lot of integration time despite of being bright. Figure 11 shows such an example. LRLL54631 is a star forming region containing very complicated structures and jets all over a large field of view. Studying such an object usually would require a lot of telescope pointings to address the spectroscopic properties all over this field. With the LUCI MOS spectrograph all those measurements can be done at once. With placing 39 individual slits all over the field on the locations of interests a single spectroscopic exposure measures velocities in the slit locations. With the ARGOS wide field correction we ensure that the individual measurements are not contaminated by surrounding flux, and we can use small slits and achieve a high spectral resolution. 


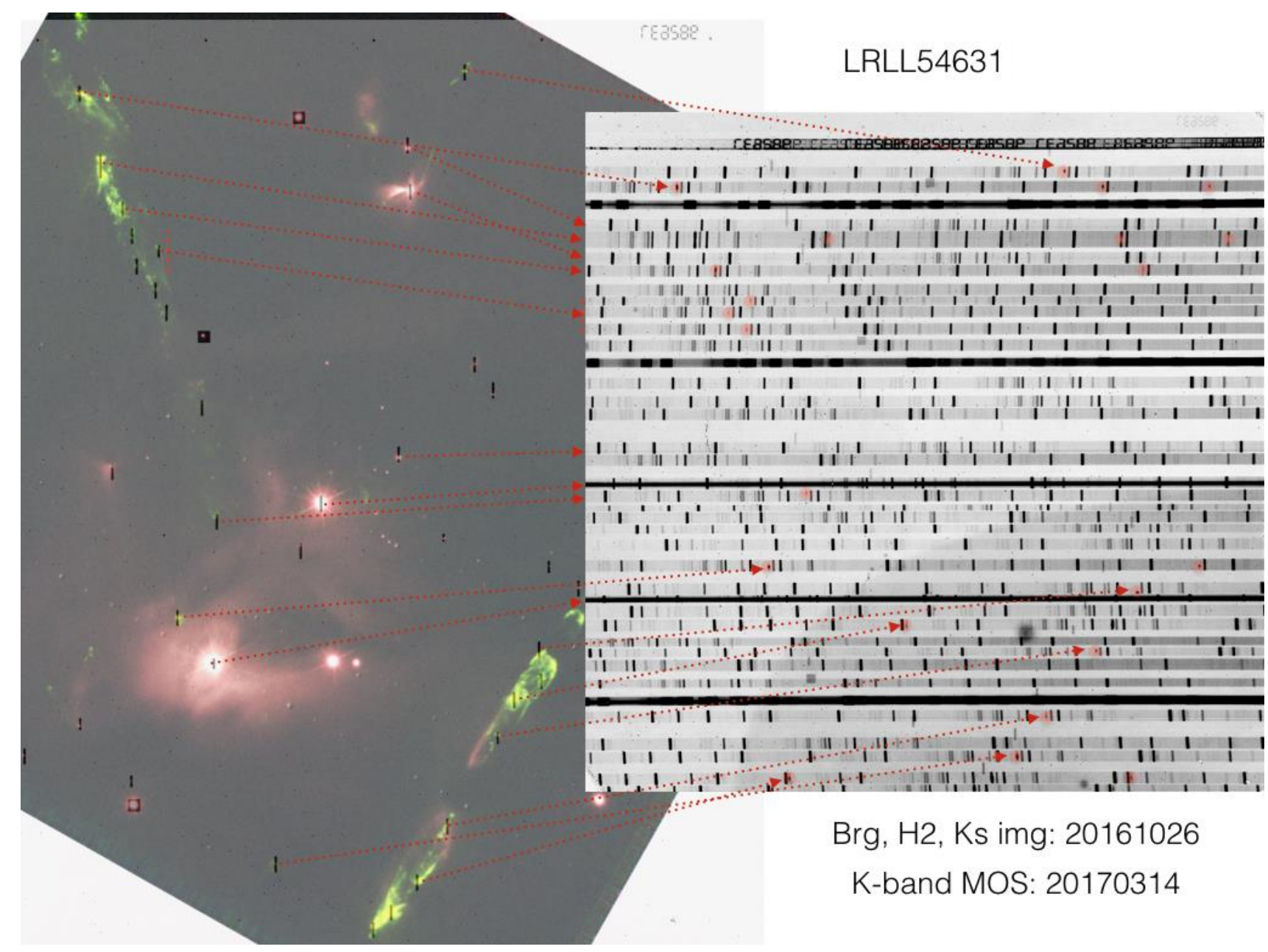

Figure 11: An example of a multi-object spectroscopic observation with LUCI ARGOS of LRLL54631 showing massive $\mathrm{H} 2$ jets whose spectroscopic properties can be measured in 39 individual locations with one observation. The high spatial resolution with ARGOS allows for small slits and thus enables a spectral resolution of $\mathrm{R}=10000$.

\section{SUMMARY}

With the commissioning of ARGOS slowly coming to an end, the system will soon be handed over for full scientific usage at the LBT. At the end of a long integration, improvement and test phase we see a system that is capable of delivering high quality scientific data. With a spatial resolution of 0.2 to 0.3 "' in the NIR we compare well with HST imaging, and can deliver high resolution spectroscopy. The first observations of imaging cases, MOS spectroscopy and custom cut slits do show the unique capabilities of this system. Even for high-z, lensed systems we already resolve details in demanding observations that are unseen with other instrumentation. 


\section{REFERENCES}

[1] Buschkamp P. et al., "LUCI2: binocular and LGS/NGS AO modes of LUCI at the LBT”, proc. SPIE 9147-58, (2014)

[2] Raab W. et al., "The ARGOS laser system: green light for ground layer adaptive optics at the LBT SPIE 9148-131, (2014)

[3] Rabien, S. et al., "Status of the ARGOS project", proc SPIE, Volume 9148, id. 91481B 13 pp. (2014)

[4] Rabien, S. et al., "ARGOS, the laser guide star system for the LBT", proc. SPIE, Volume 7736, id. 77360E-77360E12 (2010)

[5] Esposito S. et al., "Natural guide star adaptive optics systems at LBT: FLAO commissioning and science operations status", proc. SPIE 8447, (2012)

[6] Rosensteiner M. et al., "The ARGOS vibration compensation system", this proceedings

[7] Rahmer G. et al., "Early laser operations at the Large Binocular Telescope Observatory", proc. SPIE 9149-86, (2014)

[8] Orban de Xivry G., "ARGOS wavefront sensing: from detection to correction”, proc SPIE 9148-112, (2014)

[9] Bonaglia M. et al., "Pre-shipment test of the ARGOS LGS wavefront sensor", proc SPIE 9148-212, (2014)

[10] Orban de Xivry G. et al., "First on-sky results with ARGOS at LBT", proc. SPIE, Volume 9909, id. 99093610 pp. (2016)

[11] Busoni L., et al., "Commissioning of ARGOS at LBT: adaptive optics procedures", AO4ELT4, Proceedings of the conference held 25-30 October, (2015)

[12] Orban de Xivry G. et al., "First Results of the Ground Layer Adaptive Optics System ARGOS", AO4ELT4, Proceedings of the conference held 25-30 October, (2015)

[13] Shirazi M., et al. "The physical nature of the 8 o'clock arc based on near-IR IFU spectroscopy with SINFONI", Monthly Notices of the Royal Astronomical Society, Volume 440, Issue 3, p.2201-2221 (2014)

[14] Finkelstein, S. L. et al. "Turning Back the Clock: Inferring the History of the Eight O'clock Arc", The Astrophysical Journal, Volume 700, Issue 1, pp. 376-386 (2009)

[15] Dessauges-Zavadsky, M,"Nebular and global properties of the gravitationally lensed galaxy "the 8 o'clock arc" ", Astronomy \& Astrophysics, Volume 533, id.A15, 14 pp, (2011) 